\title{
Formulation and Evaluation of Novel Bilayer Floating and Sustained Release Drug Delivery System of Diltiazem HCl
}

Hoda Varasteghan ${ }^{1,2}$, Javad Shokri ${ }^{3}$, Solmaz Asnaashari ${ }^{4}$ and Yousef Javadzadeh ${ }^{1,4^{\star}}$

${ }^{1}$ Drug Applied Research Centre, Tabriz University of Medical Sciences, Tabriz, Iran

${ }^{2}$ Tabriz University of Medical Sciences, Tabriz, Iran

${ }^{3}$ Department of Pharmacy, Tabriz University of Medical Sciences, Tabriz, Iran

${ }^{4}$ Biotechnology Research Center, Tabriz University of Medical Sciences, Tabriz, Iran

*Corresponding author: Yousef Javadzadeh, Department of Pharmacy, Tabriz University of Medical Sciences, Daneshgah Street, Tabriz, Iran, Tel: +984113341315; E-mail: javadzadehy@yahoo.com

Received January 07, 2019; Accepted January 18, 2019; Published January 25, 2019

\begin{abstract}
The purpose of this study was to develop a bilayer floating drug delivery system of Diltiazem Hydrochloride (DTZ). Floating drug delivery systems can remain in the gastric region for several hours and therefore prolong the gastric residence time of drugs and improve drug bioavailability. In this study novel bilayer formulation of floating tablet comprising drug contained core and floated outer layer was prepared. The tablets were prepared by direct compression technique, using polymers such as Hydroxypropyl Methylcellulose (HPMC), Ethyl cellulose and Tragacanth alone or in combination, and other standard excipients. Sodium bicarbonate was incorporated as a gas-generating agent. Floating properties, drug release and release kinetic were assessed. The results of buoyancy and release studies showed that a combination of HPMC, tragacanth and sodium bicarbonate favors the preparation of the outer layer of bilayer floating controlled release DTZ tablets. The linear regression analysis and model fitting demonstrated that the optimized formulation followed linear probability model. Overall it was possible to control the release of highly soluble DTZ by combining the floating and bilayered tablet tools.
\end{abstract}

Keywords: Diltiazem hydrochloride; Floating; Drug delivery; Bilayer

\section{Introduction}

Diltiazem Hydrochloride (DTZ) is a calcium channel blocker. It is generally used for the treatment of hypertension and angina. DTZ goes through an extensive biotransformation, mostly through cytochrome P-450 CYP3A, which results in less than $4 \%$ of its oral dose being excreted unchanged in urine $[1,2]$. DTZ is quickly and more or less completely absorbed from the gastrointestinal tract after oral administration and the plasma half-life is 3-4 hours [3]. Extended gastric retention may be suitable for drugs that act locally like antibiotics used in the treatment of peptic ulcer. It can also be beneficial for drugs that have an absorption window in the stomach or in the upper intestine, drugs with low solubility in the intestinal tract, and for drugs that are unstable at higher $\mathrm{pH}$ [4-6]. DTZ needs multiple daily drug dosage so that it upholds adequate plasma concentrations. So, it is a proper model candidate for gastroretentive formulation [1]. Floating systems are low-density systems that have sufficient buoyancy to float over the gastric fluid and stay buoyant in the stomach without affecting the gastric emptying rate for an elongated period of time and provide a better control of the variations in plasma concentrations of the drug. Many floating systems have been established based on granules, powders, capsules, tablets, laminated films and hallow micro-spheres [7]. Floating delivery systems are designed to remain in the stomach for a long time; therefore they can reduce the variability in bioavailability that occurs with some currently available immediate and modified release systems [8]. The objective of this study was to formulate a bilayer gastro-retentive floating system and control the release rate of DTZ which has a high solubility.

\section{Materials and Methods}

\section{Materials}

Diltiazem hydrochloride was provided by Darupakhsh Co. (Tehran, Iran), Avicel (Mingtai Chemical, Bah-Der, Taiwan), Magnesium stearate (Merck), HPMC K4M (Colorcon, England), Lactose (Merck), Sodium bicarbonate (Fisher Scientific, UK), Ethyl cellulose (Aldrich Chemical Co., USA), Tragacanth (Merck).

\section{Methods}

Preparation of diltiazem floating tablets: For the first series of tablets $120 \mathrm{mg}$ of DTZ, $80 \mathrm{mg}$ of avicel and $3 \mathrm{mg}$ of magnesium stearate were mixed geometrically. The whole blend was subjected to compression using $8.00 \mathrm{~mm}$ flat faced punch on single stroke punching machine to form inner layer of our formulation. For preparing the outer layer, different amount of swellable polymers including HPMC and ethyl cellulose and tragacanth gum was added alone or in combination together and compressed using 10.00 and $12.00 \mathrm{~mm}$ flat faced punch (Figure 1). Sodium bicarbonate was incorporated as a gas-generating agent in some of the formulations. The details of formulations are summarized in Table 1 . In the next series, avicel in the inner layer was replaced with lactose and in some of the formulations a share of DTZ was blend into the outer layer formulation. The details are summarized in Table 2. Compression of all tablets was controlled to produce a 6-8 $\mathrm{kg} / \mathrm{cm}^{2}$ tablet-hardness.

Floating properties: The tablets were placed in a $500 \mathrm{~mL}$ of $0.1 \mathrm{~mol}$ $\mathrm{L}^{-1} \mathrm{HCl}$ solution. The time needed for the tablet to rise to the surface and float was taken as the floating lag time. The experiments were conducted in triplicate. Floating properties were determined using USP dissolution tester apparatus II, (Erweka, DPT6R, and Germany). Tablets were presented in the vessels in isolation and the paddles were rotating at $50 \mathrm{rpm}$ in $500 \mathrm{~mL}$ of simulated gastric fluid without pepsin $(\mathrm{pH}=1.2)$ and temperature was kept at $37 \pm 0.2^{\circ} \mathrm{C}$. The in vitro buoyancy was visually determined by floating lag times and duration of floating.

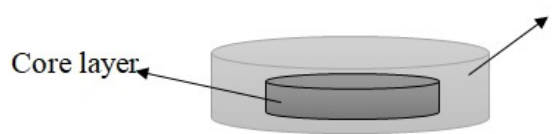

Swelling layer

Figure 1: Schematic diagram of bilayered swellable tablet system. 


\begin{tabular}{|c|c|c|c|c|}
\hline Formulation no. & HPMC $_{(\mathbf{m g})}$ & Ethylcellulose $_{(\mathbf{m g})}$ & $\mathbf{N a H C O}_{3(\mathbf{m g})}$ & Tragacanth $_{(\mathbf{m g})}$ \\
\hline F1 & 160 & - & - & - \\
\hline F2 & 240 & - & - & - \\
\hline F3 & 160 & 80 & - & - \\
\hline F4 & 200 & 40 & - & - \\
\hline F5 & 200 & 40 & 50 & - \\
\hline F6 & 200 & 40 & 25 & - \\
\hline F7 & 160 & - & - & 80 \\
\hline
\end{tabular}

Table 1: Details of outer layers of formulations 1-7.

\begin{tabular}{|c|c|c|c|c|}
\hline Formulation no. & $\mathbf{D T Z}_{(\mathbf{m g})}$ & HPMC $_{(\mathbf{m g})}$ & Tragacanth $_{(\mathbf{m g})}$ & $\mathbf{N a H C O}_{3(\mathbf{m g})}$ \\
\hline F8 & - & 160 & 80 & - \\
\hline F9 & - & 200 & 80 & - \\
\hline F10 & - & 200 & 100 & - \\
\hline F11 & 40 & 200 & 100 & - \\
\hline F12 & 60 & 220 & 110 & - \\
\hline F13 & 40 & 240 & 120 & 15 \\
\hline F14 & 60 & 200 & 100 & 15 \\
\hline
\end{tabular}

Table 2: Details of outer layers of formulations 8-14

In vitro drug release studies: Dissolution studies were performed for the formulations with acceptable floating results using USP dissolution tester apparatus II. They were carried out for 8 hours in 500 $\mathrm{mL}$ of simulated gastric fluid without pepsin as a dissolution medium under stirring speed of $50 \mathrm{rpm}$ and temperature of $37 \pm 0.2^{\circ} \mathrm{C}$, in triplicate. Samples were collected periodically and substituted with fresh dissolution medium. The withdrawn samples were filtered through a $0.45 \mu \mathrm{m}$ filter (Millipore Corp., Bedford, MA, USA) and analyzed for DTZ content at $235.6 \mathrm{~nm}$ by UV spectrophotometry (UV/visible spectrophotometer, Shimadzu-120, Japan).

Kinetic studies: To clarify the mechanism of release, the in vitro release profiles were fitted to 6 kinetic models which have been represented in Table 3.

The accuracy and prediction ability of the models were compared by calculation of squared correlation coefficients (RSQ) and absolute percent error (E) for each set as well as overall mean percent error (OE) for all sets. The values of $\mathrm{E}$ and $\mathrm{OE}$ were calculated applying the following equations:

$$
\begin{aligned}
& E=\frac{100}{N} \sum_{i=1}^{N}\left[\frac{\left|F_{c a l, i}-F_{o b s, i}\right|}{F_{o b s, i}}\right] \\
& O E=\frac{\sum_{i=1}^{18}(\mathrm{E})}{18}
\end{aligned}
$$

$\mathrm{F}_{\text {cal,i }}$ and $\mathrm{F}_{\mathrm{obs}, \mathrm{i}}$ denote calculated fraction and observed fraction of drug released at the $\mathrm{i}^{\text {th }}$ sample, respectively. The value of $\mathrm{N}$ is the number of data in each set.

\section{Results and Discussion}

F1 and F2 formulations showed proper floating lag times but their floating duration was short and after 5 hours when dissolution test was stopped, only about $40 \%$ of the drug was released. Ethyl cellulose is practically insoluble in water [9], so it was added to HPMC to prolong the floating time of the tablets (F3 and F4), but these two formulations didn't float either. Therefore, sodium bicarbonate was used as a gasgenerating agent in order to obtain floating property of the tablet (F5). Tablets floated as soon as they were placed in dissolution media and had a very short floating lag time, but the outer layer of the tablets fell apart after about 45 minutes and did not remain intact. Lowering the amount of sodium bicarbonate and also increasing of HPMC were useful for extending the duration of floating a little but was not desirable value
(F6), therefore they were omitted from dissolution studies. For the next step, HPMC was mixed with tragacanth, which is a natural gum that when it is mixed with water, merely the soluble part, called tragacanthin, dissolves to provide a colloidal hydrosol while the insoluble part swells to form a gel. It was added to keep the integrity of HPMC for a longer time (F7). Tragacanth gum was a proper choice for prolonging the time of buoyancy. F7 formulation was buoyant for over 8 hours but the tablets had very poor release rate in the first 6 hours. To solve this problem, avicel in the core of tablets was substituted with lactose which is a stronger disintegrate. Although this substitution increased the drug release (Figure 2) but it shortened the floating duration almost by half. Gradually increasing the amount of HPMC and tragacanth extended the floating duration (F9 and F10) but still there was problem with drug release especially in the first hours. To overcome this issue, it was suggested that a fraction of the drug could be mixed with the outer layer components. So $1 / 3$ of DTZ was combined with HPMC and tragacanth (F11). There was an increase of drug release in the first few hours but buoyancy time was very short. Increasing the amount of polymers did not help us for obtaining proper floating time extension.

Next step for resolving this matter was adding a small amount of sodium bicarbonate in order to initiate the floating. Adding of about $15 \mathrm{mg}$ of $\mathrm{NaHCO}_{3}$ to the mixture of HPMC and tragacanth aided with decreasing the floating lag time to 15 minutes. Tablets remained buoyant for about 6 hours and the pores formed by dissolving of $\mathrm{NaHCO}_{3}$, helped increased drug release from floating tablets. Besides, increasing the amount of DTZ in the outer layer gave a more linear release rate

\begin{tabular}{|c|c|c|c|c|c|}
\hline \multirow{3}{*}{ Zero order } & $k_{0}$ & 0.0018 & \multirow{3}{*}{$\begin{array}{c}\text { Three seconds root of } \\
\text { mass }\end{array}$} & $k_{2 / 3}$ & 0.0015 \\
\hline & RSQ & 0.9829 & & RSQ & 0.9619 \\
\hline & E & 13.8594 & & $E$ & 25.8128 \\
\hline \multirow{3}{*}{ First order } & $K_{f}$ & 0.0041 & \multirow{4}{*}{ Weibull } & $\beta$ & 1.0223 \\
\hline & RSQ & 0.8801 & & $t_{d}$ & 364.728 \\
\hline & E & 74.0309 & & RSQ & 0.9328 \\
\hline \multirow{3}{*}{ Higuchi } & $\mathbf{k}_{\mathrm{H}}$ & 0.0459 & & $E$ & 18.6168 \\
\hline & RSQ & 0.9209 & \multirow{4}{*}{ Linear probability } & $\mathbf{Z}_{0}$ & -1.5508 \\
\hline & E & 36.8285 & & $q$ & 0.0057 \\
\hline \multirow{4}{*}{ Power law } & $k_{p}$ & 0.005 & & RSQ & 0.9869 \\
\hline & p & 0.812 & & E & 9.9976 \\
\hline & RSQ & 0.9784 & \multirow{4}{*}{ Log-probability } & $\mathbf{Z}_{0}$ & -4.0837 \\
\hline & $E$ & 10.6623 & & $q^{\prime}$ & 0.7552 \\
\hline \multirow{3}{*}{ Hixson-Crowell } & $\mathbf{k}_{1 / 3}$ & 0.001 & & RSQ & 0.8477 \\
\hline & RSQ & 0.9277 & & $E$ & 29.6335 \\
\hline & $E$ & 44.2231 & \multirow{4}{*}{$\begin{array}{c}\text { Reciprocal powered } \\
\text { time }\end{array}$} & $\mathbf{m}$ & 1.006 \\
\hline \multirow{3}{*}{$\begin{array}{c}\text { Square root of } \\
\text { mass }\end{array}$} & $k_{1 / 2}$ & 0.0013 & & b & 1.2323 \\
\hline & RSQ & 0.9465 & & RSQ & 0.865 \\
\hline & E & 34.025 & & $E$ & 471.244 \\
\hline
\end{tabular}
(Figures 3 and 4).

Table 3: Kinetic results of F29 formulation.

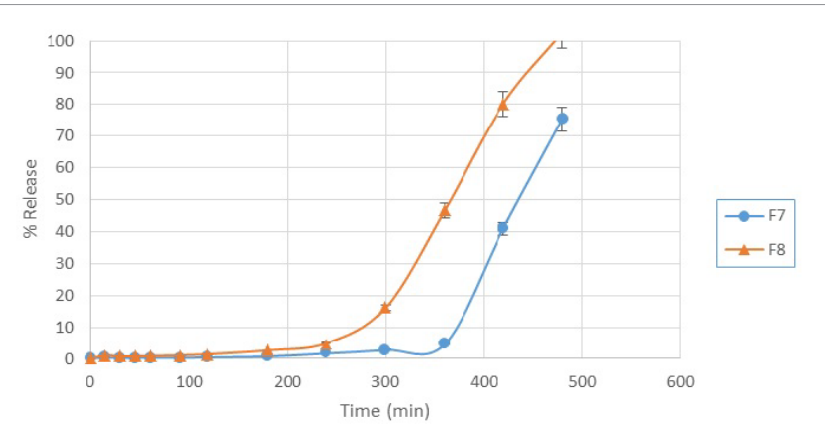

Figure 2: The effect of alternating avicel (F7) with lactose (F8) in the core. 


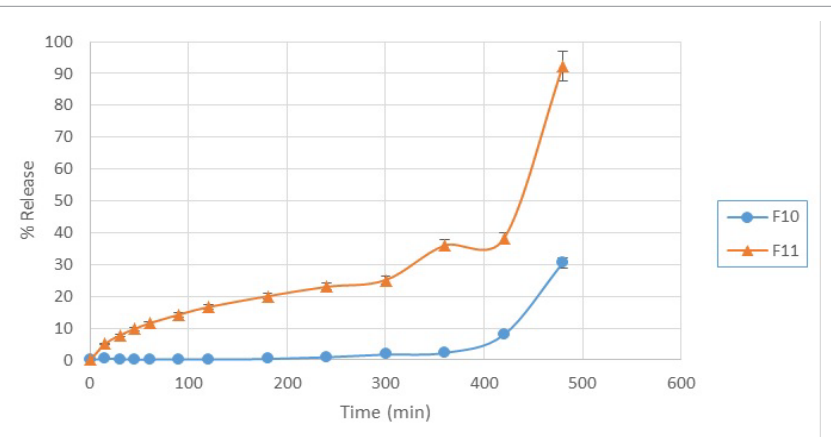

Figure 3: The effect of adding 1/3 of DTZ with components of outer layer (F11) in comparison with no DTZ in outer layer (F10).

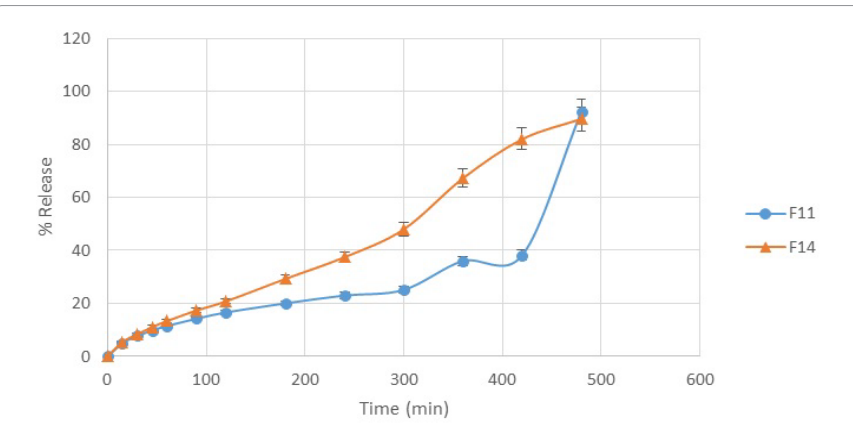

Figure 4: The effects of adding $\mathrm{NaHCO}_{3}$ and $1 / 2$ of DTZ to the outer layer (F14) in comparison with adding $1 / 3$ of DTZ and no $\mathrm{NaHCO}_{3}$ (F11).

Linear regression analysis and model [10] fitting showed that the optimized formulation followed linear probability which had higher value of correlation coefficient, RSQ. Accordingly, the release of DTZ was controlled by linear probability dissolution model. HPMC and tragacanth swell and form a gel layer upon contact with water and then slowly wear away. Water-soluble drugs are released by diffusion through the swollen layer. While soaking, the tablet exterior hydrates to form a gel layer which swells with time. With time, swelling of the polymer causes enhances the diffusional path length of the drug and lowers the drug release rate. Later, as the polymer is more diluted, it loses its structural integrity and goes through corrosion. A combination of both these effects leads the drug release in a controlled behavior which follows a linear probability release rate [4].

\section{Conclusion}

In the present research work, gastro-retentive tablets were formulated using DTZ, Lactose, HPMC, tragacanth gum and sodium bicarbonate. It was possible to control the release of highly soluble DTZ by combining the floating and bilayered tablet technologies. Drug release was found to occur by diffusion through swelling. It was mostly controlled by the polymer swelling which was a little influenced by gasgenerating agent. Consequently, by altering the amount of components in formulations, the characterization of tablets could be controlled. This system can probably be used for controlling the release of other highly water-soluble drugs.

\section{Acknowledgments}

The Researchers would like to thank the National Institute for Medical Research Development (NIMAD) for financial supports of this study (Grant Number: 943639)

\section{References}

1. Gambhire MN, Ambade KW, Kurmi SD, Kadam VJ, Jadhav KR (2007) Development and in vitro evaluation of an oral floating matrix tablet formulation of diltiazem hydrochloride. AAPS Pharm Sci Tech 8: 166-E174.

2. Adibkiaa KH, Shokrib J, Barzegar-Jalalia M, Solduzian M, Javadzadeh Y (2014) Effect of solvent type on retardation properties of diltiazem $\mathrm{HCl}$ form liquisolid tablet. Colloids and Surfaces B: Biointerfaces 113: 10-14.

3. Patel AN, Patel FM, Rathore KS (2011) Formulation and characterization of floating tablets of Diltiazem hydrochloride. J Pharma Biomed Sci 9: 2230-7885.

4. Desai RS, Rohera BD (2014) Formulation, in vitro evaluation and study of variables on tri-layered gastro-retentive delivery system of diltiazem $\mathrm{HCl}$. Drug Dev Ind Pharma 40: 380-389.

5. Adibki KH, Hamedeyazdan S, Javadzadeh Y (2011) Drug release kinetics and physicochemical characteristics of floating drug delivery systems. Expert Opin Drug Deliv 8: 891-903.

6. Javadzadeh $\mathrm{Y}$, Hamedeyazdan $\mathrm{S}$, Adibkia $\mathrm{KH}$, Kiafar $\mathrm{F}$, Zarrintan $\mathrm{MH}$, et al (2010) Evaluation of drug release kinetics and physicochemical characteristics of metronidazole floating beads based on calcium silicate and gas-forming agents. Pharma Dev Tech 15: 329-338.

7. Vengatesh S, Elango K, Damayanthi RD, Deattu N, Christina P (2012) Formulation and evaluation of floating tablets of ondansetron hydrochloride. Int J Drug Dev Res 4: 264-274.

8. Asnaashari S, Khoei NS, Zarrintan MH, Adibkia K, Javadzadeh Y (2011) Preparation and evaluation of novel metronidazole sustained release and floating matrix tablets. Pharma Dev Tech 16: 400-407.

9. Javadzadeh Y, Monajjemzadeh F, Safaei E, Adibkia KH (2013) Release Kinetics of Sodium Diclofenac from a Controlled Release Device. Pharm Ind 75: 1-12.

10. Azharshekoufeh L, Shokri J, Barzegar-Jalali M, Javadzadeh Y (2017) Liquigroud technique: a new concept for enhancing dissolution rate of glibenclamide by combination of liquisolid and co-grinding technologies. Bio Impacts 7: 5-12. 